\title{
Silent Ride (SIRI)
}

\author{
Adhithya P.P1, Ayana P Dharman², Lishmi M.D ${ }^{3}$, Sreejith P.S ${ }^{4}$ \\ B. Tech Student, Department of Computer Science and Engineering, Universal Engineering College, Vallivattom, \\ Kerala, India ${ }^{1,2,3}$
}

Associate Professor, Department of Computer Science and Engineering, Universal Engineering College, Vallivattom,

Kerala, India ${ }^{4}$

\begin{abstract}
Vehicle to Vehicle Communication is the type of communication which provides communication among nearby vehicles for reducing sound pollution. Traffic is increasing day by day and still we cannot save ourselves with this increasing traffic. With the help of this technology we can take one step towards to reducing sound pollution and accidents. This technology is called vehicle-to vehicle communication because one vehicle is communicating with the other vehicle or we can say one vehicle is sending its information to other vehicle. The communication is done with the help of RF communication. This system also detects the accident and sent the current location to the nearest hospital by using piezo sensor. This paper offers a brief introduction of various communication technologies and different features used in vehicle-to-vehicle communication.
\end{abstract}

Keywords: Vehicle to Vehicle Communication; RF Communication; hit detection; RF tag reading; Data transmission

\section{INTRODUCTION}

In the urban areas most of the sound pollution are caused because of noisy environment which is created by industrial machineries and vehicle horn. Among all the reasons the vehicle horn is assumed most vital for making the noisy environment which has a hazardous impact on the human health. Therefore, it is become graver to need a system that is able to make the environment safe and sound. To confront this challenge, this study proposes a V2V or Vehicle to Vehicle Communication System. In this study the vehicle to vehicle communication has been accomplished using network and RF reader without using horn. The system contains software and hardware. This system reduces sound pollution in the environment. This communication is done via mobile application and RF reader. The vehicle should be on the application when the vehicle starts and driver must use it for easy checking when moving the vehicle. RF reader sense the vehicle in front of the moving vehicle then generate a unique ID of the RF tag for identifying that vehicle then the mobile application send the message to the nearby vehicle by using this unique ID. This unique ID used for identifies the vehicles. All these processes are done by pressing the buzzer. This system also detects the accident and sent the current location to the nearest hospital by using the piezo sensor according to the threshold value of hit.

\section{LITERATURE SURVEY}

In ${ }^{[1]}$ vehicle sound is probably the most rigorous and pervasive source of sound pollution among other sources such as neighborhood, electrical appliances, TV and music systems, public address systems, railway and air traffic and electricity generating sets. Most of the vehicle noise are generated in traffic congestion which is caused by unnecessary honking of vehicle horn. In most cases, driver irresponsibility is the main reason behind the vehicle noise. Driver creates a hazardous situation by honking unnecessarily in traffic jam which adversely effect in the human body. Researchers have found vehicle noise pollution to be a major cause of several human defects like stress, unborn and effects on hearing. Over the past few years researchers adopt some system that could mitigate the vehicle noise in some extent. As computerized technology advances, there is a push towards Vehicle-to vehicle Communication. Though vehicle to vehicle communication is mainly used for traffic safety but this study presented a system which can be used as a substitute of vehicle horn. In this system RF and IR communication has been used as a replacement of vehicle horn. Every vehicle has been attached with a RF and IR Transmitter-Receiver pair where RF transmitter and IR Transmitter are used to send the signal with a message to the nearest vehicle.

In ${ }^{[2]}$ this paper provides knowledge on Radio Frequency Identification (RFID) technology. Initially RFID tags were made to eventually replace barcodes in different chains. Their advantages are that they can be read wirelessly and with no line of sight, contain more data than barcodes, and are stronger. As the paper describes the recent technology, include the frequency ranges used and standards required. With the increase in ubiquity of RFID tags, however, privacy became unease. The paper outlines probable attack that can go against one's privacy and it also describes contradict 
measures. The RFID technology did not stop at thing-level tagging. Since the uses for RFID tags are so extensive, there is a large interest in lowering the costs for production of RFID tags. It turns out that printing tags may become a possible alternative to traditional production.

$\mathrm{In}^{[3]}$ Automatic accident detection focuses on increasing the safety, automatic accident detection, emergency assistance and road hazard warning dissemination. Internet of things for smart cities addresses an urban IoT system that is specific to an application domain. Urban IoT, supports the Smart City vision, providing most advanced communication technologies and services to the citizens.Using Smartphone to detect car accidents and provide situational awareness to Emergency response provides solutions to the major problems in detecting traffic accidents to avoid false positives and increases preparedness to emergency responders.

In ${ }^{[4]}$. Vehicles are able to communicate with each other in Vehicle to Vehicle (V2V) or with Vehicle to Road Communication (V2R). Some of the application vehicle networks are security distance warning, vehicle collision warning, map location, driverless vehicle etc.. most of the application need traffic speed and travel time calculation. This calculation helps to road way users to identify which routes to use. This calculation can be saved to analyze traffic speed and travel time for different time interval. Speed based algorithm which seems to have false alarm when sudden brake are applied. In fact vehicle accidents are the most common cause of death more than cancer or heart attack. If you think about serious accident, it could change your life. So this paper helps to protect the people from the accident by the use of GSM, GPS, and MEMS.

In ${ }^{[5]}$ this paper concentrated on low speed car accident detection. The main obstacle that encounters the low speed accident is how to differentiate whether the user is inside the vehicle or outside the vehicle, walking or slowly running. The effect of this obstacle is minimized, in this work, by a proposed mechanism that distinguishes between the speed variation of low speed vehicle and walking or slowly running person. The proposed system consists of two phases; the detection phase which is used to detect car accident in low and high speeds. The notification phase, and immediately after an accident is indicated, is used to send detailed information such as images, video, accident location, etc. to the emergency responder for fast recovery.

In ${ }^{[6]}$ this system uses the RFID technology where RFID tag is attached to each vehicle and as and when vehicle reach the traffic junction the RFID reader will read those RFID tags. Depending upon the count of vehicles green passage will be set dynamically and the proposed system provides special privileges for emergency vehicles like police vehicle, ambulance, VIP vehicles, etc. RFID also enforces law against stolen vehicles. System uses the RFID technology where RFID tag is attached to each vehicle and as and when vehicle reach the traffic junction the RFID reader will read those RFID tags. Depending upon the count of vehicles green passage will be set dynamically and the proposed system provides special privileges for emergency vehicles like police vehicle, ambulance, VIP vehicles, etc. RFID also enforces law against stolen vehicles.

In ${ }^{[7]}$ large numbers of personal vehicles adapted by the society. Due to such advent road accident is one of the serious problems of the age. It is necessary to provide safety to automobile systems in adverse conditions. To track vehicle accident's location GPS system is used and for reporting GSM modem is utilized. In this system, we have used piezo disk to sense the collision during accident. The microcontroller reads along voltage value from sensor and digitizes it. When threshold value of sensor is exceeded, corresponding GPS coordinates of accident location are sent to the predefined numbers via GSM. If the driver is safe, he/she needs to turn OFF safety switch manually to prevent message sending. This system will be beneficial to manufacture low-cost safety system for automobiles.

\section{SYSTEM OVERVIEW}

\section{A) Software Requirements}

1. Firebase Database: Firebase can power app's backend, including data storage, user authentication, static hosting, and more. Focus on creating extraordinary user experiences. We will take care of the rest. Build cross-platform native mobile and web apps with our Android, iOS, and JavaScript SDKs.

2. Android Studio: Android is an open source software package that was developed primarily to improve the mobile user experience and has now become the best-selling operating system for mobile devices. Android is now a part of Google and the Open Handset Alliance, and the current version of the Android operating system is Oreo (version 8.0).

3. Java Script: Java Server Pages is a technology that helps software developers create dynamically generated web pages based on HTML, XML, or other document types.

4. XML: Extensible Markup Language is a markup language that defines a set of rules for encoding documents in a format that is both human-readable and machine-readable. The W3C's XML 1.0 Specification and several other related 
Vol. 8, Issue 4, April 2019

specifications all of them free open standards. The design goals of XML emphasize simplicity generally, and usability across the internet. It is a textual data format with strong support via Unicode for different human languages. Although the design of XML focuses on document, the language is widely used for the representation of arbitrary data structure such as those used in web services.

\section{B) Hardware Requirements}

1. RF reader: An RFID reader's function is to identify RFID tags. A reader contains a Radio Frequency module, which acts as both a transmitter as well as receiver of radio frequency signals. The transmitter consists of an oscillator to create the carrier frequency. The receiver has a demodulator to extract the returned data and also for processing it contains an amplifier to strengthen the signal. A microprocessor forms the control system, memory to filter and store the data. The data is then ready to be sent to the network.

2. RF tag: An RFID tag is a smooth card of credit-card size which is read by an RFID reader. It works at $125 \mathrm{kHz}$ and comes with a unique 32-bit ID. Normally, each tag has a unique ID number which cannot be changed.

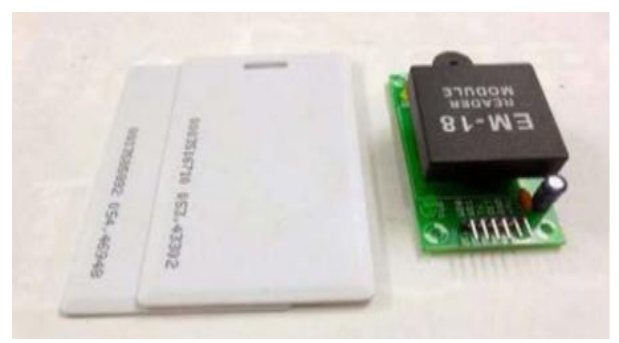

Fig : RF reader and RF tag

3. Piezo Sensor: A piezoelectric sensor is a device that uses the piezoelectric effect, to measure changes in pressure, acceleration, temperature, strain or force by converting them to an electrical charge.

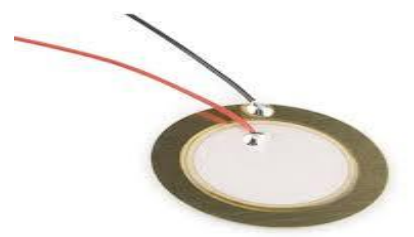

4. Node MCU: NodeMCU is like brand name of a board that has a wifi module ESP8266 and some associated circuit. ESP8266 module in tune has a micro controller with wifi.

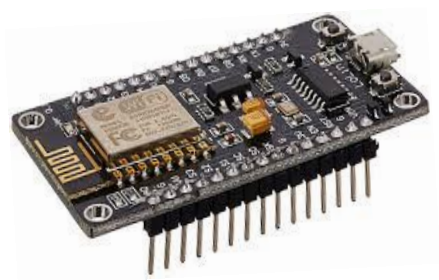

5.GPS module: The GPS receiver gets a signal from each GPS satellite. The satellites transmit the exact time the signals are sent. So given the travel time of the GPS signals from three satellites and their exact position in the sky, the GPS receiver can determine your position in three dimensions - east, north and altitude.Here when a hit occurred between the vehicle then the current location will be sent to the nearest hospital through GPS.

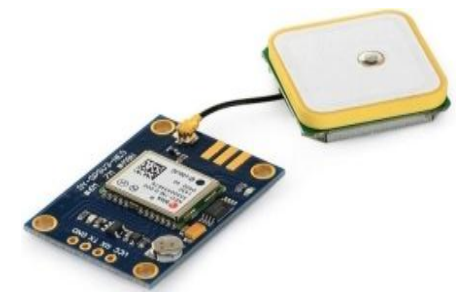


C) Block diagram

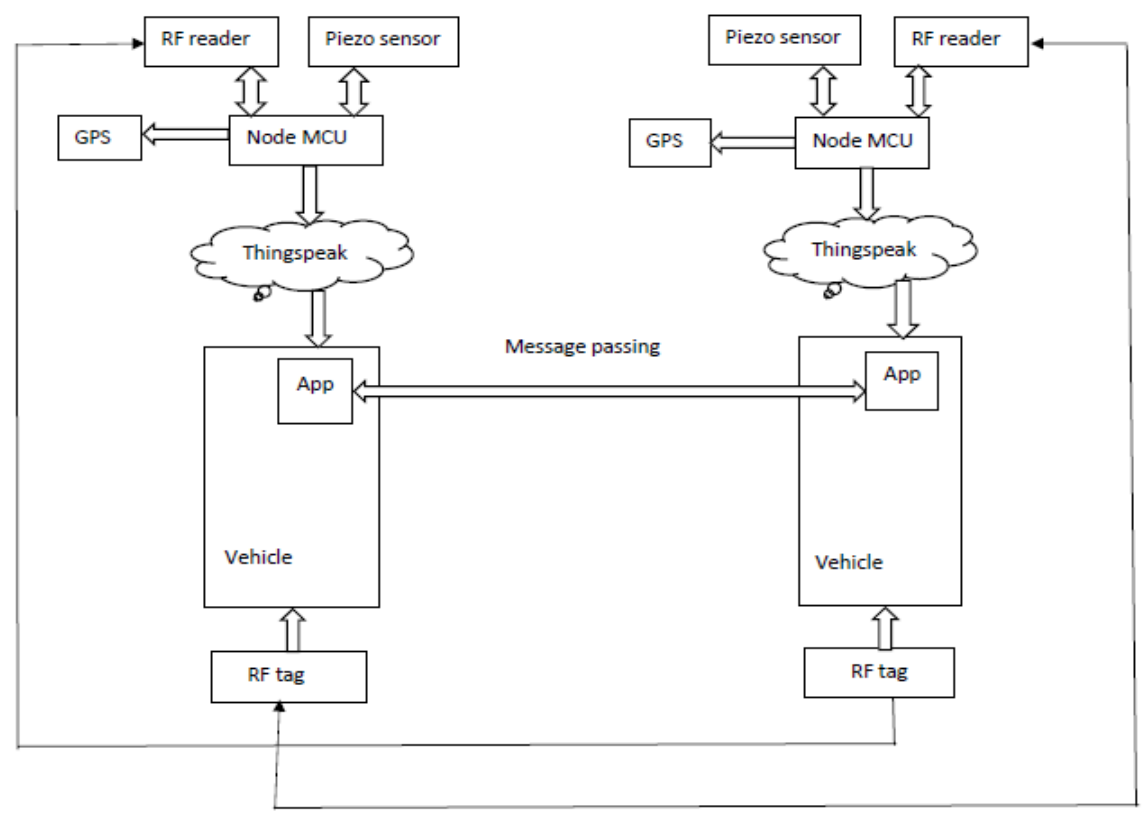

Fig: Block diagram

In this system the RF reader reads the unique ID from the RF tag and using this unique id the mobile application exchange messages without honking between vehicles in traffic areas. All these are controlled by a NODEMCU. In some situations likes curves and turning the The RF reader will not be able to identify other vehicle so, normal horn will be produced. The accident detection is done by using the piezo sensor. Here we already set a threshold value 50 in the piezo sensor if there is any hit is detected a value is generated by the piezo sensor then compare the value with the threshold value. if hit greater than 50 then, send a warning message with the current location to nearest hospital through GPS. Otherwise, the message is discarded.

D) Data Flow Diagram

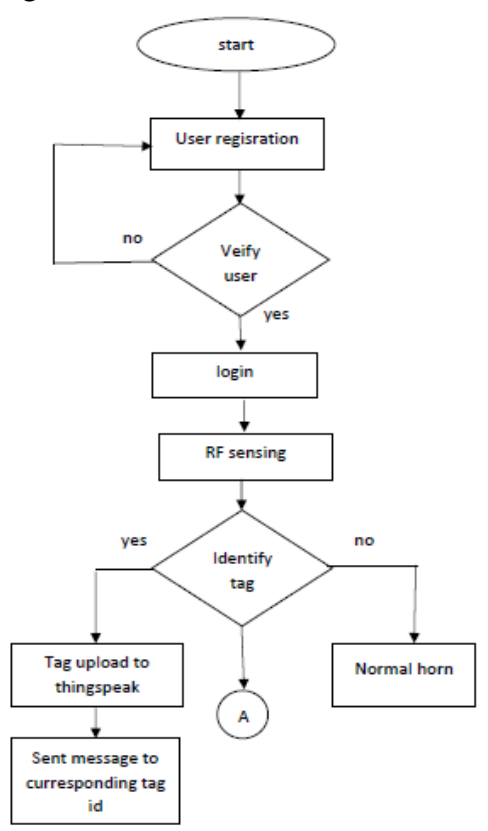

Fig : User registration and RF sensing

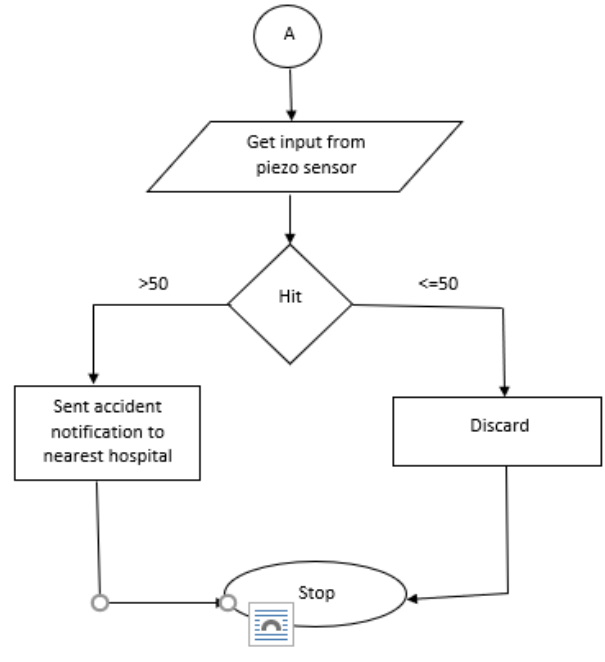

Fig :Accident detection 


\section{METHODOLOGY}

This RF communication and accident detection consists of various micro-controller, software and hardware components. There is various methodologies used in this system.

\section{A) Data-capture technology}

This system uses RF module and mobile application to exchange message without honking between vehicles in traffic area. The RF module consists of RF reader and RF tag. The RF reader is attached in the front side of the every vehicles and the RF tag is attached to the back side of the every vehicles. The RFID reader has a radio transmitter and receiver inside. It is also called as an interrogator. The RF reader reads the unique ID from the RF tag and using this unique id the mobile application exchange messages without honking between vehicles in traffic areas. It contain mainly two scenario:

1. First and second scenario

2. The first scenario is RF reader reads the unique ID from the RF tag and using this unique id the mobile application exchange messages without honking between vehicles in traffic areas. The second scenario is situation likes curves and turns, the normal horn will be provided. The RF reader will not be able to identify other vehicle.

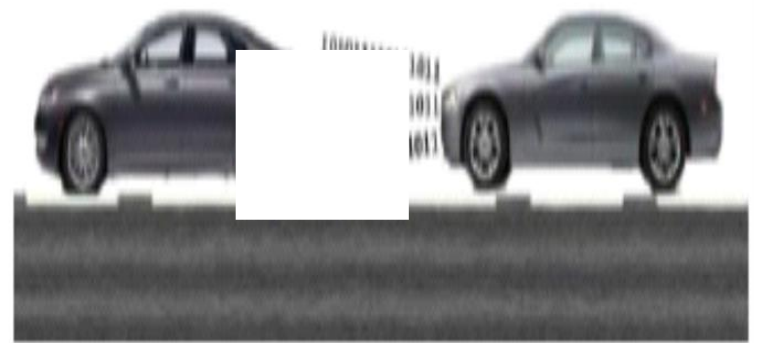

Fig : First scenario

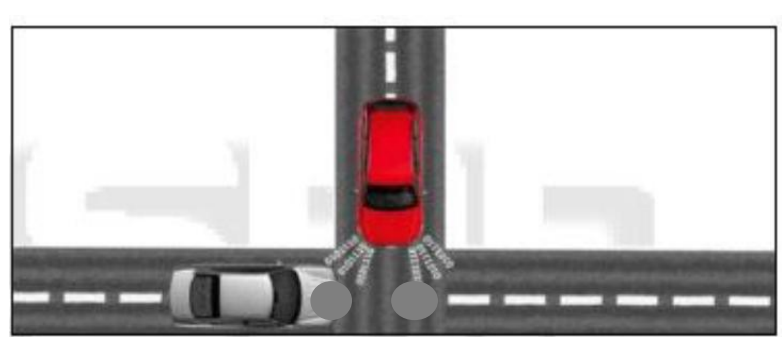

Fig :Second scenario

\section{Reading distance}

Read range refers to the maximum distance at which RFID reader can detect signal from the tag. The RF reader is attached in the front side of the every vehicles and the RF tag is attached to the back side of the every vehicles and RF reader read unique id from $\mathrm{RF}$ tag in particular distance.

\section{B) Mobile interference}

The message passing is done by using the mobile application installed every car driver's handset. The nodeMCU microcontroller contains the wifi module and using this wifi module the mobile application is connected with the device. After connecting the device, the user registration is done by user's gmail id and tag id and the registrations details are stored in the firebase database. When we start the vehicle the mobile application is also open. The RF reader identifiy the vehicle moved in front of our vehicle, then the RFID module capture the tag id of the nearest vehicle and compare the id with the tag id's already stored in the database. If the Id is matched the data transmission through this mobile application without honking between the vehicles in traffic area.

\section{User Registration}

The mobile application aims at providing a one-time registration form for capturing user's unique id and the corresponding datas. The personal data include: (a) Name, (b) Email, (c) tag ID. The whole record of users' information is uploaded to firebase database once the registration process is complete.

\section{SMS notification}

The RF reader identify the vehicle moved in front of our vehicle, then the RFID module capture the tag id of the nearest vehicle and compare the id with the tag id's already stored in the database. If the Id is matched, the data transmission is done through this mobile application without honking between the vehicles in traffic areas. The message is send to the nearest vehicle's user id and the message is shown in the user's handset.

\section{c) Accident Detection}

A piezoelectric sensor is a device that uses the piezoelectric effect, to measure changes in pressure, acceleration, temperature, strain, or force by converting them to an electrical charge. If any hit occurred between two vehicles then 
piezo sensor will be activated at the moment and the hit value is measured by the piezo sensor, then compare the value with the threshold value 50. If the value is higher than the threshold value a warning message or accident declaration message is send to the nearby hospital through GPS with current location.

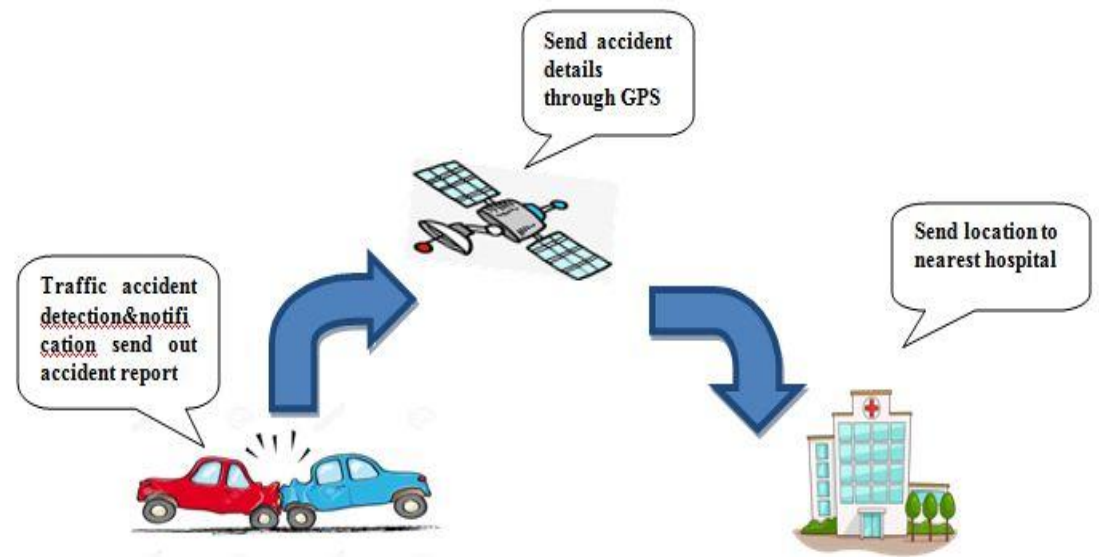

Fig: Accident detection through GPS

\section{HIGH/LOW hit}

The accident detection is done by using the piezo sensor. Here we already set a threshold value 50 in the piezo sensor if there is any hit is detected a value is generated by the piezo sensor then compare the value with the threshold value. if hit greater than 50 then, send a warning message with the current location to nearest hospital through GPS. Otherwise, the message is discarded

\section{Notification phase}

If the hit is detected, the value generated by the piezo sensor is compared with the Threshold-value and send a warning message and current location to the nearest hospital's web server through GPS. Then the message is shown in the hospitals web server and they can take the emergency actions as soon as possible.

\section{RESULTS AND COMPARISONS}

The proposed system reduces sound pollution and communicates with the vehicles without making any external horn. Instead of sending message wirelessly among the vehicles. This message is displaying in an mobile handset by the mobile application used in this system. So that the driver could take movement step based on the received message. And also detect accidents occurred in the traffic areas and send message to the nearest hospitals server. So the hospital's authority can take emergency action as soon as possible.

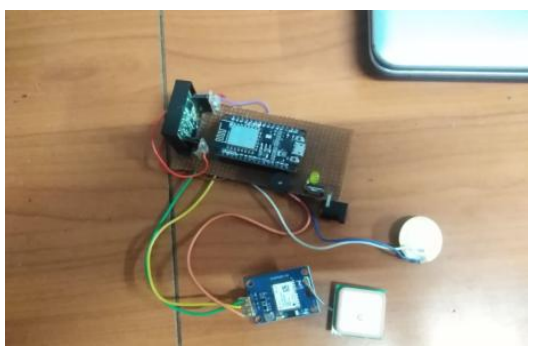

It also provides many advantages compared to traditional systems, namely, minimizing sound pollution, reduce human diseases, recognizing exact and accurate accidents locations, and providing medical facilities.Total performance better than traditional system because This project provides very good idea of how we can extract a location of accident and send the SMS notifications to nearest hospital and sensor provide better working and accuracy.

\section{CONCLUSION AND FUTURE WORK}

This paper has presented a brief review on reduce sound pollution and accident detection techniques. These techniques include V2V communication, RF communication, smart phones, GPS technologies and mobile applications. These 
Vol. 8, Issue 4, April 2019

systems aim to timely inform emergency services about the location of accident, in the process of saving precious lives. The main aim of this system is to making sound pollution free environment. So that the people are not be affected by various diseases which caused by unnecessary vehicle horn. For future improvement, this system will use the mobile network for the message passing. In some cases the mobile network is not accessed in some regions here we can improve this system without using the mobile network. Then the other improvement is we can extend the use of this system in two wheelers also with the help of smart helmets.

\section{ACKNOWLEDGMENT}

It is our immense pleasure to work on this Project, Design of Vehicle to Vehicle Data Transmission .It is only the blessing of my divine master which has prompted and mentally equipped me to undergo the study of this Project. We are thankful to professor Dr. Vince Paul, Head of Computer Science Engineering Department for his precious encouragement at every phase of our Project Work. I offer my sincere thanks to my guide Prof. Sreejith P.S affectionately encourages me to work on the subject and gave her valuable guidance time to time. The proposed system can reduce the sound pollutions and the it can detect the detect the accidents occurred the traffic areas as soon as possible and save the value of human life.

\section{REFERENCES}

[1]. Eftekhar Hossain, Nursadul Mamun, Md.Faisal, Vehicle to Vehicle Communication Using RF and IR Technology, 2nd International Conference on Electrical \& Electronic Engineering (ICEEE), 27-29 ,December 2017.

[2]. Dhrubajyoti Adak, Manoj Kumar Pain, Uttam Kumar Dey, RFID BASED SECURITY SYSTEM USING ARDUINO MODULE, International Journal of Scientific \& Engineering Research, vol Volume 8, Issue 3, March-2017.

[3]. Spurti Shinde, Shweta Joshi, Nikita Shah, Shweta Tatiya, Preeti Kumari, Accident Detection and Alert Systems for Immediate Emergency Services: A Literature Review, International Journal of Science and Research, 2319-7064.

[4]. P.Kaliuga Lakshmi, C.Thangamani, AN EFFICIENT VEHICLE ACCIDENT DETECTION USING SENSOR TECHNOLOGY, International Journal of Advanced Research in Computer Engineering \& Technology, Volume 5 Issue 3, March 2016.

[5]. Hamid M. Ali1, Zainab S. Alwan, Car Accident Detection \& Notification System Using Smartphone, IJCSMC, Vol.4, Issue.4, April 2015, pg. $620-635$.

[6]. Priyanka Nalawade, Prajakta Waghere, Nisha Vanare, Prajakta Kalbhor, A. J. Jadhav, Dynamic Traffic Control System using RFID Technology: A Systematic Review, International Journal of Advanced Research in Computer and Communication Engineering, Vol. 6, Issue 1, January 2017,pg 66-68.

[7]. Rohit Ganiga, Rohit Maurya , Archana Nanade, Accident Detection System Using Piezo Disk Sensor, International Journal of Science, Engineering and Technology Research,, Volume 6, Issue 3, March 2017,356-359.

[8]. Aarti Chauhan, Nidhi Sharma, Vehicle-To-Vehicle Communication: Traffic Safety Over RF Communication, International Journal of scientific research and management, Volume 3, Issue 5,Pg 2769-2777,2015.N.

[9]. M. Z. Hashim, A. S. Jaafar, N. A. Ali, L. Salahuddin, N. R. Mohamad, M. A. Ibrahim, Traffic Light Control System for Emergency Vehicles Using Radio Frequency, IOSR Journal of Engineering, Vol. 3, Issue 7 ,July. 2013, PP 43-52.

[10]. Maudhoo Jahnavi, Neha Yadav, Krishanu Griyagya, Mahendra Singh Meena, Ved Prakash, Vehicle to Vehicle Communication for Collision Avoidance, International Journal for Research in Applied Science \& Engineering Technology, Volume 6 Issue V, May 2018.

[11]. GOH CHIA CHIEH, DINO ISA, Low cost approach to real-time vehicle to vehicle communication using parallel CPU and GPU processing, International Journal of Advanced Computer Science and Applications, Vol. 3, No. 12, 2012,pg 33-43.

[12]. Mrs. Vaishali D. Khairnar Dr. S.N. Pradhan, V2V COMMUNICATION SURVEY - (WIRELESS TECHNOLOGY), Vaishali D Khairnar et al, Int.J. Computer Technology \& Applications, Vol 3 (1), 370-373,jan 2012.

[13]. Adithya B, Vehicle to Vehicle Wireless Communication Protocol, International Journal of Science and Research, Volume 4 Issue 3, March 2015,1341-1351.

[14]. Mr. Jagdish A. Patel, Sagar C. Dighe, Shubhangi S. Shinde, Yashwant N. Memane4, Suvarna B.Mahajan, Li-Fi Technology-Vehicle to Vehical Data Transmission, INTERNATIONAL JOURNAL OF INNOVATIVE RESEARCH IN ELECTRICAL, ELECTRONICS, INSTRUMENTATION AND CONTROL ENGINEERING Vol. 4, Issue 4, April 2016 ,215-217.

[15]. Paul Golding and Vanesa Tennant, Evaluation of a Radio Frequency Identification (RFID) Library System: Preliminary Results, International Journal of Multimedia and Ubiquitous Engineering Vol. 3, No. 1, January 2008,1-16.

[16]. .Mandeep Kaur, Manjeet Sandhu, Neeraj Mohan and Parvinder S. Sandhu, RFID Technology Principles, Advantages, Limitations \& Its Applications, International Journal of Computer and Electrical Engineering, Vol.3, No.1, February, 2011,pg 152-157.

[17]. Pranali More1, Ulhas Patil2, Prof. Avinash Ingole, Survey on Accident Detection, Tracking and Recovery of Vehicles, International Research Journal of Engineering and Technology, Volume: 04 ,Issue: 10 , Oct -2017,pg 1942-1945.

[18]. Usman Khalil and Tariq Javid, Adnan Nasir, Automatic Road Accident Detection Techniques :A Brief Survey, Conference Paper November 2017

[19]. B.Rani, R. Praveen Sam, Govardhan Reddy Kamatam, A Review on Vehicle Tracking and Accident Detection System using Accelerometer, International Journal of Applied Engineering Research ISSN 0973-4562 Volume 13, Number 11 (2018) pp. 9215-9217.

[20]. Prof. Chethana Gosal, Akshay Chadaga P, Anush Balraj R, Madhu Kiran K M, Manoj T M, Intelligent Automatic Vehicle Accident Detection and Prevention System, Prof. Chethana Gosal.et.al. Int. Journal of Engineering Research and Application, Vol. 7, Issue 7, ( Part -2) July 2017, pp. $37-47$ 